\title{
Inserção e atuação do profissional de educação física nos núcleos de apoio à saúde da família em Santos-SP1
}

\section{The insertion and performance of the physical education professional in the family health support centers in Santos-SP}

\section{Inserción y desempeño del profesional de educación física en los centros de apoyo a la salud familiar de Santos-SP}

(iD) Luciana Maria de Sobral

Universidade Federal de São Paulo - campus Baixada Santista, Santos, São Paulo, Brasil e-mail: luciana_jcv@hotmail.com

iD (9) Rogério Cruz de Oliveira

Universidade Federal de São Paulo - campus Baixada Santista, Santos, São Paulo, Brasil e-mail: rogerio.cruz@unifesp.br

iD (9) Ricardo José Gomes

Universidade Federal de São Paulo - campus Baixada Santista, Santos, São Paulo, Brasil e-mail: ricardojosegomes@yahoo.com.br

iD Camila Aparecida Machado de Oliveira

Universidade Federal de São Paulo - campus Baixada Santista, Santos, São Paulo, Brasil e-mail: cam.oliveira@unifesp.br

Resumo: O objetivo deste estudo foi compreender a inserção e a atuação do profissional de Educação Física no NASF em Santos-SP. Trata-se de uma pesquisa de abordagem qualitativa, na qual participaram 3 profissionais de Educação Física. A coleta de dados se deu por entrevista semiestruturada, e os dados foram interpretados à luz da análise de

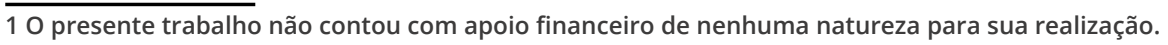


conteúdo. Como resultado, compreende-se que a inserção dos profissionais no NASF é inerente ao cargo ocupado. A atuação se dá num cenário limitante em número de profissionais e legitimação, trabalho anterior isolado e uma prática pedagógica instrumental, entretanto, aberta aos ideários críticos. Assim, embora haja um potencial alinhamento da atuação em relação às diretrizes do NASF, conclui-se que ainda existem limites a superar.

Palavras-chave: Promoção da Saúde. Atenção Primária à Saúde. Práticas Corporais. Atividade Física.

Abstract: The aim was to comprehend the insertion and performance of the Physical Education professional in the Family Health Support Centers (NASF) in the city of Santos-SP. It is a qualitative research in which three Physical Education professionals participated as volunteers. For this, semi-structured interviews were carried out and the data were analyzed through content analysis. As a result, the insertion of these professionals in the NASF is inherent to the position held. The performance is developed in a limiting context in terms of number of professionals and legitimacy, previous separate work, and an instrumental pedagogical practice; however, it opens to critical framework. Although the performance is potentially aligned with the NASF, we concluded there are limits to surpass yet.

Keywords: Health Promotion. Primary Health Care. Body Practices. Exercise.

Resumen: El objetivo de este estudio fue conocer la inserción y desempeño del profesional de Educación Física en el NASF en SantosSP. Se trata de una investigación cualitativa, en la que participaron 3 profesionales de la educación física. La recolección de datos se realizó a través de entrevistas semiestructuradas y los datos se interpretaron a la luz del análisis de contenido. Como resultado, se entiende que la inserción de profesionales em el NASF es inherente al cargo que ocupan. La actuación se desarrolla en un escenario que limita el número de profesionales y la legitimidad, un trabajo previo aislado y una práctica pedagógica instrumental, sin embargo, abierta a ideas críticas. Así, 
aunque existe una potencial alineación de desempeño en relación con las directrices del NASF, se concluye que aún quedan límites por superar. Palabras-clave: Promoción de la Salud. Atención Primaria a la Salud. Prácticas Corporales. Actividad Física.

Submetido em: 09-12-2020

Aceito em: 17-02-2021 
Inserção e atuação do profissional de educação física nos núcleos de apoio à saúde... Luciana Maria de Sobral • Rogério Cruz de Oliveira • Ricardo José Gomes • Camila Aparecida Machado de Oliveira

\section{Introdução}

O Núcleo de Apoio à Saúde da Família (NASF)2 foi criado mediante a Portaria 154/2008 (BRASIL, 2008) a fim de aumentar o escopo de ações e resolubilidade na Atenção Básica a Saúde (ABS). Um NASF deve ser constituído por uma equipe, na qual profissionais de diferentes áreas de conhecimento atuam em conjunto com os profissionais das equipes de Saúde da Família (eSF), compartilhando e apoiando as práticas em saúde nos territórios sob responsabilidade delas. Tal composição deve ser definida pelos próprios gestores municipais e as equipes das unidades eSF, mediante critérios de prioridades identificadas a partir das necessidades locais e da disponibilidade de profissionais de cada uma das diferentes ocupações. O NASF não se constitui porta de entrada do sistema para os usuários, mas sim de apoio às eSF (BRASIL, 2010).

O NASF faz parte da ABS, mas ele não se constitui como um espaço físico independente, pois, para ele, utilizam-se os espaços das Unidades Básicas de Saúde (UBS) e do território para a realização do seu trabalho, inserindo-se no âmbito da ABS para oferecer apoio às eSF (BRASIL, 2014). Ele também faz parte da Rede de Atenção à Saúde (RAS), e, para que esta rede de apoio atinja positivamente os usuários, é necessário um trabalho intersetorial. "A intersetorialidade é essa articulação entre sujeitos de setores sociais diversos e, portanto, de saberes, poderes e vontades diversos, a fim de abordar um tema ou situação em conjunto" (BRASIL, 2010, p.18), com equipes multiprofissionais atuando interprofissionalmente. Para que isso ocorra, é preciso uma relação de trabalho mais colaborativa, a qual requer intensa articulação entre as várias categorias de profissionais para uma produção de cuidado mais integralizada e mais efetiva (BRASIL, 2014, p. 39).

O Sistema Único de Saúde (SUS) tem reformulado suas intervenções, implantando novas perpectivas visando uma rede de cuidado com mais integralidade (MAGALHÃES JÚNIOR, 2014). Nesse

2 Em 2017, o NASF virou NASF-AB (Núcleo Ampliado de Saúde da Família e Atenção Básica), mas, por questões de identificação, adotaremos apenas NASF ao longo do trabalho. 
Inserção e atuação do profissional de educação física nos núcleos de apoio à saúde... Luciana Maria de Sobral • Rogério Cruz de Oliveira • Ricardo José Gomes • Camila Aparecida Machado de Oliveira

processo, existe o argumento de que as práticas corporais/atividades físicas (PCAF) podem ser reconhecidas como fator de proteção contra riscos que ameaçam a saúde (KOKUBUN et al., 2007), evidenciando a importância da atuação do Profissional de Educação Física (PEF) nos serviços de saúde, em conjunto com uma equipe multiprofissional.

Entretanto, o advento da Política Nacional de Promoção da Saúde (PNPS) - indutora da inserção dos PEF's no SUS, ampliou o seu campo de atuação, o que, para Bueno (2016), colocou a profissão em destaque. Ainda para a autora, o NASF criou uma possibilidade de atuação do PEF na saúde na perspectiva do cuidado centrado no usuário, para além do fator de proteção contra o risco da doença. Nesse sentido, sua prática não se limita à beleza estética, indo além "[...] do aperfeiçoamento da aparência física, estando associados à melhora da saúde física e mental" (SCHUH et al., 2015, p. 30). Para Carvalho (2006, p.36), as PCAF têm na ABS um espaço importante de atenção à saúde do usuário, podendo ser ressignificada por processos educativos voltados à construção de vínculos, "[...] de responsabilidades, para a autonomia (individual e coletiva) e para a capacidade de provocarem a mudança do pensar e agir em saúde", ao encontro da cultura, no caso da educação física (EF), a cultura corporal. O Ministério da Saúde preconiza que o PEF em sua atuação deve favorecer

[...] a diversidade das manifestações da cultura corporal presentes localmente e as que são difundidas nacionalmente, procurando fugir do aprisionamento técnico pedagógico dos conteúdos clássicos da Educação Física, seja no campo do esporte, das ginásticas e danças, bem como na ênfase à prática de exercícios físicos atrelados à avaliação antropométrica e à performance humana (BRASIL, 2010, p. 126).

Frente ao exposto, há de reconhecer a importância desse campo de atuação que se abriu para a EF nos últimos 10 anos e buscar compreendê-lo à luz das normativas vigentes e outras ex- 
Inserção e atuação do profissional de educação física nos núcleos de apoio à saúde...

Luciana Maria de Sobral • Rogério Cruz de Oliveira • Ricardo José Gomes • Camila Aparecida Machado de Oliveira

periências. Assim, o objetivo do estudo consiste em compreender a inserção e a atuação do profissional de Educação Física no NASF em Santos-SP.

\section{Método}

Trata-se de um estudo exploratório-descritivo, que, para Triviños (2008), respectivamente, se ocupa em aumentar a experiência dos/as pesquisadores/as em determinado problema, bem como busca se ocupar dos fatos e fenômenos de determinada realidade. Nesse sentido, é a abordagem qualitativa que mais se aproxima dos pressupostos metodológicos do estudo, pois ela trabaIha com um universo de dados não quantificáveis, se ocupando de "[...] significados, motivos, aspirações, crenças, valores e atitudes [...] que não podem ser reduzidos à operacionalização de variáveis" (MINAYO, 1994, p.21-22).

O estudo foi aprovado pelo Comitê de Ética da Universidade Federal de São Paulo sob CAAE n 08917118.1.0000.5505.

Participaram do estudo 3 PEF's que atuam no NASF da Secretaria Municipal de Saúde de Santos-SP (SMS), o que compreende o número total desses profissionais. Após autorização da SMS, o recrutamento foi feito pessoalmente nos respectivos locais de trabalho. Todos assinaram um Termo de Consentimento Livre e Esclarecido.

A produção de dados se deu por entrevista semiestruturada, que, para Trivinõs (2008), parte de certos questionamentos básicos que interessam à pesquisa, permitindo ao informante a liberdade e espontaneidade necessárias à investigação, sendo uma das principais ferramentas de pesquisas com abordagens qualitativas. Para Minayo (2014), trata-se de uma conversa com finalidade, que, no caso deste estudo, além do perfil dos voluntários (idade, sexo, formação e tempo de atuação na SMS e NASF), obedeceu ao seguinte roteiro: 
Inserção e atuação do profissional de educação física nos núcleos de apoio à saúde... Luciana Maria de Sobral • Rogério Cruz de Oliveira • Ricardo José Gomes • Camila Aparecida Machado de Oliveira

- NASF: entendimento, trabalho pré e pós-NASF;

- Prática Pedagógica33: Público-alvo; frequência e duração das atividades; objetivos; conteúdo/conhecimentos trabaIhados; metodologia, e; avaliação;

- Trabalho em equipe.

As entrevistas foram realizadas nos meses de junho e julho de 2019 numa única oportunidade com cada voluntário, sendo desenvolvidas em ambiente reservado. Com o consentimento dos voluntários, as entrevistas foram gravadas e, posteriormente, transcritas para um arquivo eletrônico. Com vistas a preservação da identidade, os voluntários foram identificados por letras e números.

A interpretação dos dados se deu por análise de conteúdo das falas dos entrevistados, sendo consideradas as fases descritas em Campos (2004): pré-exploração, a qual consiste em apreender os aspectos importantes do material por meio de leitura exaustiva; seleção das unidades de análise por meio de recortes de frases e/ ou parágrafos trascritos que fazem sentido ao objetivo do estudo; e, por fim, a categorização, que são "[...] grandes enunciados que abarcam um número variável de temas [...] que atendam os objetivos do estudo [...]" (p.614). No caso deste estudo, utilizamos a categorização não apriorística, que se forma a partir do contato dos pesquisadores com o material analisado.

\section{Resultados}

De acordo com o Quadro 1, é possível observar que se trata de PEF's com uma década de atuação na saúde e com especialização diretamente ligada à respectiva atuação profissional.

\footnotetext{
3 Para Bracht (1992), toda prática pedagógica precisa responder a duas questões: o porquê (sentido) e o como (instrumento). Para Freitas (1995), a prática pedagógica se espraia nos pares dialéticos da didática: objetivos $\mathrm{x}$ avaliação; conteúdo $\mathrm{x}$ método. Embora os autores se pronunciem sobre uma prática pedagógica do campo da educação escolar, acredita-se que tais preceitos podem ser úteis ao campo da promoção da saúde e ao NASF, justamente por lidar com práticas educativas.
} 
Inserção e atuação do profissional de educação física nos núcleos de apoio à saúde... Luciana Maria de Sobral • Rogério Cruz de Oliveira • Ricardo José Gomes • Camila Aparecida Machado de Oliveira

\section{Quadro 1 - Perfil dos voluntários}

\begin{tabular}{|c|c|c|c|c|c|c|}
\hline Voluntários & Sexo & Idade & G & PG & $\begin{array}{c}\text { TASMS } \\
\text { (anos) }\end{array}$ & $\begin{array}{c}\text { TANASF } \\
\text { (anos) }\end{array}$ \\
\hline PEF1 & M & 40 & $\begin{array}{c}\text { Licenciatura Plena em } \\
\text { Educação Física (2004) }\end{array}$ & $\begin{array}{c}\text { Especialização em } \\
\text { Apoio a Saúde da } \\
\text { Família }\end{array}$ & 10 & 4 \\
\hline PEF2 & M & 41 & $\begin{array}{c}\text { Licenciatura Plena e Ba- } \\
\text { charelado em Educação } \\
\text { Física (2003) }\end{array}$ & $\begin{array}{c}\text { Especialização em } \\
\text { Apoio a Saúde da } \\
\text { Família }\end{array}$ & 10 & 4 \\
\hline PEF3 & F & 44 & $\begin{array}{c}\text { Licenciatura Plena em } \\
\text { Educação Física (2004) }\end{array}$ & $\begin{array}{c}\text { Especialização em } \\
\text { Apoio a Saúde da } \\
\text { Família }\end{array}$ & 10 & 4 \\
\hline
\end{tabular}

Fonte: dados da pesquisa (2020).

M: masculino; F: feminino; G: graduação; PG: pós-graduação; TASMS: tempo de atuação na SMS; TANASF: tempo de atuação no NASF.

No que se refere ao trabalho na saúde, todos os voluntários afirmaram não ter tido vivência pregressa, nem mesmo em sua formação profissional.

Não tínhamos vivência prática na área da saúde [...] fomos conhecer a EF na saúde através do trabalho. PEF1

[...] eu vinha de uma trajetória escolar. Assim que me formei eu trabalhei em uma escola, trabalhei em uma instituição de menores (lá na antiga FEBEM, que agora é Fundação (asa). Aí vinha com essa bagagem. PEF2

[...]a gente nunca foi trabalhado com matérias específicas da área de saúde. Então, fui aprender a trabalhar com saúde na saúde [...]. PEF3

Cabe destacar ainda que os voluntários realizaram concurso público junto à Secretária Municipal de Esportes do município de Santos-SP (SME), sendo remanejados para atuação na SMS no momento da admissão, como mencionado pelo PEF3: [...] fiz o concur- 
Inserção e atuação do profissional de educação física nos núcleos de apoio à saúde...

Luciana Maria de Sobral • Rogério Cruz de Oliveira • Ricardo José Gomes • Camila Aparecida Machado de Oliveira

so para trabalhar na Secretaria de Esportes [...] a gente só foi descobrir que era para a Secretaria de Saúde nos exames admissionais [...].

Sobre a inserção no NASF, os voluntários afirmaram que se deu por razão de sua implantação na SMS em 2015. Para a composição das equipes, foram convidados profissionais que já exerciam suas funções na SMS. Todos os PEF's foram convidados a fazer um curso de Especialização em Apoio a Saúde da Família - concluído pelos 3 voluntários (vide Quadro 1) -, sendo uma das formas de aproximação desse profissional com o NASF.

Cada PEF foi designado para atuação em um NASF de um território específico, a saber:

- PEF 1: Morros - com 7 eSF;

- PEF 2: Centro - com 7 eSF;

- PEF 3: Zona Noroeste - com 6 eSF.

O município de Santos-SP ainda possui um $4^{\circ}$ território (Orla), mas, até o momento da coleta de dados, ainda não possuía equipe NASF.

No que se refere ao entendimento do NASF (ENASF), o Quadro 2 nos permite identificar a categoria "Melhora do Trabalho na ABS", seja pela implementação das equipes, matriciamento ou cuidado para os munícipes. Já em relação ao trabalho pré-NASF desenvolvido pelos PEF's (TpNASF), há a identificação da categoria: “Exercício Físico para o Hiperdia", fato esse que limitava o trabalho dos PEF's (Quadro 2). Por fim, em relação ao trabalho no NASF (TNASF), foi possível identificar duas categorias de análise: "Ampliação do escopo de atuação do PEF" e "Melhor vínculo com o munícipe" (Quadro 2). 
Inserção e atuação do profissional de educação física nos núcleos de apoio à saúde... Luciana Maria de Sobral • Rogério Cruz de Oliveira • Ricardo José Gomes • Camila Aparecida Machado de Oliveira

\section{Quadro 2 - NASF na perspectiva dos voluntários}

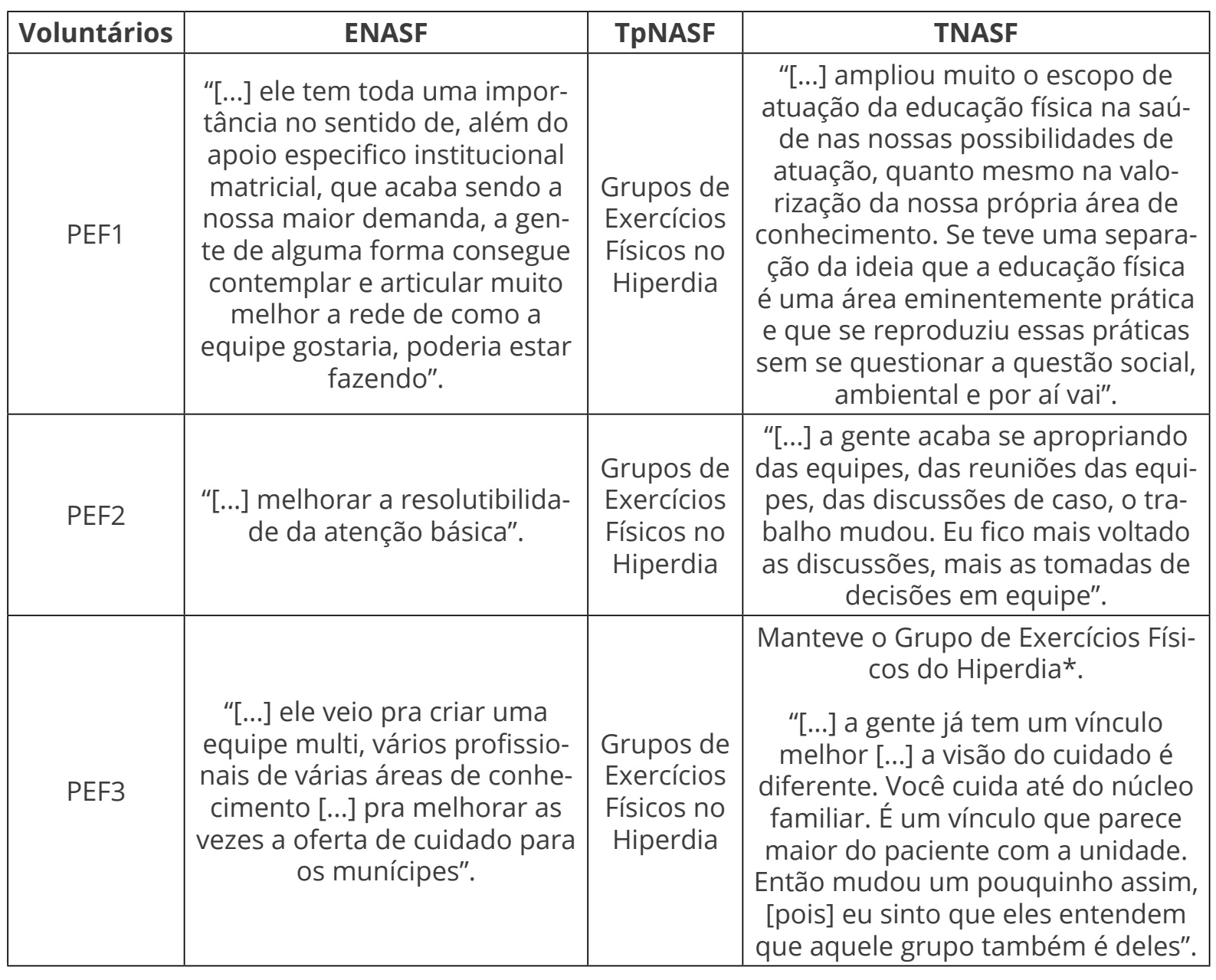

Fonte: dados da pesquisa (2020).

No contexto da prática pedagógica, o Quadro 3 demonstra que o discurso se alinha às práticas de caminhada, alongamento e fortalecimento muscular na esteira do cuidado com pessoas idosas com doenças crônicas, sendo a avaliação incipiente. 
Inserção e atuação do profissional de educação física nos núcleos de apoio à saúde... Luciana Maria de Sobral • Rogério Cruz de Oliveira • Ricardo José Gomes • Camila Aparecida Machado de Oliveira

\section{Quadro 3: Prática Pedagógica dos PEF's no NASF}

\begin{tabular}{|c|c|c|c|c|c|}
\hline Voluntários & PA & OB & CON & MET & AVA \\
\hline PEF1 & $\begin{array}{c}\text { Mulheres idosas } \\
\text { com polipatolo- } \\
\text { gias. }\end{array}$ & $\begin{array}{c}\text { Convivência. } \\
\text { Promoção } \\
\text { da saúde. }\end{array}$ & $\begin{array}{c}\text { Fortaleci- } \\
\text { mento mus- } \\
\text { cular, alon- } \\
\text { gamentos, } \\
\text { estímulos } \\
\text { propriocep- } \\
\text { tivos. }\end{array}$ & $\begin{array}{c}\text { Aquecimento, } \\
\text { parte principal } \\
\text { e volta à calma. }\end{array}$ & $\begin{array}{c}\text { Anamnese } \\
\text { anual }\end{array}$ \\
\hline PEF2 & $\begin{array}{c}\text { Mulheres idosas } \\
\text { com doenças } \\
\text { crônicas. }\end{array}$ & $\begin{array}{c}\text { Melhorar as } \\
\text { condições de } \\
\text { vida diária. }\end{array}$ & $\begin{array}{c}\text { Flexibilidade } \\
\text { e força. } \\
\text { Caminhada. }\end{array}$ & $\begin{array}{c}\text { Aquecimento, } \\
\text { parte principal } \\
\text { e volta à calma. }\end{array}$ & Não há \\
\hline PEF3 & $\begin{array}{c}\text { Pessoas idosas } \\
\text { com doenças } \\
\text { crônicas. }\end{array}$ & $\begin{array}{c}\text { Desenvolver } \\
\text { autonomia. }\end{array}$ & $\begin{array}{c}\text { Mobilidade } \\
\text { e equilíbrio. } \\
\text { Caminhada. }\end{array}$ & $\begin{array}{c}\text { Aquecimento, } \\
\text { parte principal } \\
\text { e volta à calma. }\end{array}$ & Não há \\
\hline
\end{tabular}

Fonte: dados da pesquisa (2020).

PA: público-alvo; OB: objetivo (s); CON: conteúdo/conhecimento; MET: metodologia; AVA: avaliação.

Em termos de número de participantes, os grupos variavam de 5 a 60 munícipes, sendo as atividades ofertadas numa frequência de 2 vezes semanais em sessões de 1 h de duração. Entretanto, os PEF's também citaram o desenvolvimento de trabalhos com adolescentes em contexto escolar (PEF1 e PEF3), gestantes (PEF1) e agentes comunitários de saúde (PEF1). Nesse contexto, os conteúdos/conhecimentos desenvolvidos, respectivamente, foram: atividade física, violência e sexualidade; trabalho postural e parto; e corpo. Nesses demais grupos, as reuniões são mensais e possuem características de palestras, de orientações relacionadas à prática de atividade física, promoção e prevenção à saúde num contexto geral.

Os PEF's relataram ainda participar das reuniões das eSF e também do NASF. As reuniões são semanais com dias e horários fixos, mas reuniões adicionais podem ocorrer sempre que necessário. Para os PEF's, as reuniões são momentos de trocas de conhecimento, discussão de casos, planejamento de ações e cronogramas 
Inserção e atuação do profissional de educação física nos núcleos de apoio à saúde... Luciana Maria de Sobral • Rogério Cruz de Oliveira • Ricardo José Gomes • Camila Aparecida Machado de Oliveira

das atividades que serão realizadas e/ou eventos. São uma espécie de eixo norteador que arquiteta todas as ações desenvolvidas nas comunidades das UBS de referência. Embora para o PEF3 esse seja ainda um momento desafiador, pois: "[...] o professor de $E F$ parece uma pessoa inquieta, ele gosta de se mexer, ele gosta de fazer, ele gosta de extravasar e ficar sentando duas ou três horas [numa reunião é difícil] [...] eu preciso me encontrar nesse novo momento".

Nesse sentido, é possível compreender o discurso sobre a prática pedagógica dos PEF's no NASF em duas categorias: "Prática pedagógica tradicional" e "Prática pedagógica emancipatória". A primeira é aquela voltada aos estímulos físicos, aos munícipes envolta por uma metodologia tradicional de trabalho, enquanto a segunda se mostra ocupada pela convivência, melhoria das condições de vida e autonomia.

Já em relação ao trabalho em equipe, os voluntários foram unânimes em afirmar que isso melhorou a possibilidade de compreensão da saúde e de sua própria intervenção, configurando a categoria "Avanço na atuação". No entanto, ainda relatam fragilidades. Para PEF1, às vezes, o trabalho em equipe numa perspectiva interprofissional não se concretiza por inteiro: "[...] a nossa equipe, às vezes fica um pouco frustrada, mas não por não conseguir fazer, mas por querer fazer mais". Já para PEF2, a esteira é outra, se refere à legitimidade da EF na saúde: "[...] as profissões [ainda] não entendem claramente o que que a gente pode fazer [...] então a gente tem muito que conquistar espaço". Já para PEF3, o trabalho ainda está caminhando e pode melhorar, pois todos estão em aprendizado, principalmente em relação a entender "[...] como é que também dá para trabalhar junto".

\section{Discussão}

Em Souza e Loch (2011), percebem-se algumas semelhanças com os dados do nosso estudo em relação à formação dos profis-

\footnotetext{
4 Na direção de Kunz (1994), para o qual a emancipação é o processo que possibilita às pessoas o uso da razão crítica nos processos educacionais frente as demandas da sociedade.
} 
Inserção e atuação do profissional de educação física nos núcleos de apoio à saúde... Luciana Maria de Sobral • Rogério Cruz de Oliveira • Ricardo José Gomes • Camila Aparecida Machado de Oliveira

sionais, principalmente na ausência de conhecimentos de saúde pública e investimento em formação continuada para lidar com as demandas do trabalho.

Entretanto, ressalta-se que a inserção do PEF na área da saúde é um fenômeno relativamente recente, pois é a partir dos anos 2000 que há um aumento das possibilidades de sua atuação na ABS (LOCH, DIAS e RECH, 2019, p.1). Devido à pouca experiência prática na área, esses profissionais enfrentam grandes desafios para consolidar a sua atuação junto ao SUS (SANTOS e BENEDETTI, 2012). Para Fonseca et al. (2012), o cenário de formação em EF para a saúde no Brasil ainda é recente, entretanto, destaca duas universidades pioneiras: o curso de EF da Universidade Federal de São Paulo - Campus Baixada Santista, implantado no ano de 2006, e o curso de EF e Saúde da Universidade de São Paulo (Escola de Artes, Ciências e Humanidades), em 2005. Para Oliveira e Andrade (2016), trata-se de um desafio, haja vista que a formação em EF ainda possui outras áreas de intersecção como o esporte, o lazer e a educação. O desafio ainda é maior devido ao distanciamento das Diretrizes Curriculares Nacionais dos cursos de graduação em Educação Física (DCNEF) (BRASIL, 2018a) do debate acumulado sobre o SUS e, ainda, do descompasso com as Diretrizes Curriculares Nacionais dos cursos de graduação da área da saúde (BRASIL, 2018b), a qual, por exemplo, afirma o trabalho em equipe como um princípio de formação, sequer mencionado pelas DCNEF.

Em relação à atuação no NASF, Santos e Benedetti (2012) afirmam que sua expansão no Brasil significou um aumento na proporção de profissionais atuando na área da saúde, sendo o PEF uma das cinco categorias mais recrutadas. Dessa maneira, a inserção desses profissionais aumentou o seu campo de atuação, ampliando o escopo de ações dentro de uma equipe multiprofissional, corroborando o discurso dos voluntários do estudo.

Nessa esteira, Loch, Dias e Rech $(2019$, p.5) afirmam que a atividade física tem um papel importante, não só em direção 
Inserção e atuação do profissional de educação física nos núcleos de apoio à saúde... Luciana Maria de Sobral • Rogério Cruz de Oliveira • Ricardo José Gomes • Camila Aparecida Machado de Oliveira

[...] à doença e aos agravos crônicos em saúde, mas também por seu aspecto cultural e sua potência de contribuir para a mudança do modelo de atenção, privilegiando um olhar ampliado de saúde, coerente com o princípio da integralidade inclusive, em detrimento ao olhar biomédico, ainda hegemônico.

Para Assis et al. (2010), a inversão desse modelo de atenção é de difícil execução, pois exige articulação com uma política de formação aliada à prática, sendo necessária a formação de uma rede de apoio e atenção à saúde do usuário, "[...] em que trabalhadores, gestores e usuários devam ser corresponsáveis no fazer saúde, cotidianamente" (p.14). Assim, o que os PEF's desse estudo trazem sobre a possibilidade da melhora de vínculo com os usuários - um importante fator para promover a integralidade do cuidado - demonstra alinhamento com esse ideário de transformação do modelo de atenção, corroborando a PNAB (BRASIL, 2012). O vínculo consiste em uma construção de afetividade e confiança entre usuário e trabalhador da saúde, permitindo o aprofundamento do processo de corresponsabilização pela saúde, o que é adquirido com o tempo.

Para os PEF's, o NASF veio para melhorar a relação de trabaIho, impulsionando assim o campo específico da EF. Entretanto,

\begin{abstract}
[...] pensar e agir em saúde numa perspectiva de mudança do modelo requer um novo modo de estabelecer relações e troca de saberes e experiências práticas, envolvendo campos de conhecimento e intervenção interdisciplinares (ASSIS et al., 2010, p.14).
\end{abstract}

Ou seja, significa conviver diariamente com incertezas, frustrações, dúvidas, recorrência à tradição da área no que se refere ao exercício físico e sua relação de causa-consequência com a saúde, mas também ousadias em direção a temas latentes nas comunidades, tais como gravidez, parto, violência, corpo etc. Nesse sen- 
Inserção e atuação do profissional de educação física nos núcleos de apoio à saúde... Luciana Maria de Sobral • Rogério Cruz de Oliveira • Ricardo José Gomes • Camila Aparecida Machado de Oliveira

tido, é possível afirmar que o discurso dos PEF's sobre sua atuação no NASF "pendula" entre um saber fazer tradicional, um saber fazer emancipatório e um saber fazer com outros saberes (não específicos à área, mas caros ao campo da saúde). Tais possibilidades acabam por conferir um terreno fértil, porém árduo de atuações. Isso porque a formação profissional em EF, como já discutido, ainda está distante da produção de cuidado em saúde e suas possibilidades.

Em relação ao atendimento aos usuários, Saporetti, Miranda e Belisário (2016) e Souza e Loch (2011) encontraram dados semelhantes ao nosso estudo, no qual a maioria dos usuários nos grupos eram idosos e possuíam alguma patologia, tais como diabetes e hipertensão. A quantidade de usuários que participavam dos grupos variava entre 5 a 60 a cada grupo, observando-se semelhança com a pesquisa de Souza e Loch (2011). Observa-se aqui um cenário limitante de atuação do PEF no NASF, principalmente quando se compreende que deve haver o acolhimento das diversas faixas etárias de munícipes com ou sem patologias. Por outro lado, faz-se necessário compreender um histórico pregresso de atuação dos PEF's na SMS com esses grupos e o ínfimo número desses profissionais atuantes no NASF, que, no nosso entendimento, carece de extensa ampliação. Além disso, existe uma ampla atuação dos PEF's no contexto da SME, com mais de 80 profissionais concursados desenvolvendo atividades em 3 Centros Esportivos e na Orla da cidade. Tais fatores parecem esvaziar as ações dos PEF's no NASF em direção a outros grupos e práticas, sendo passível de investigações em outros estudos. Koumantareas e Oliveira (2018), por exemplo, que investigaram as concepções de esporte e saúde para PEF's atuantes na SME, afirmam a existência de uma polissemia relacionada ao esporte e à saúde no contexto investigado, podendo denotar práticas pedagógicas distintas. Se esse é o cenário no contexto da formação esportiva, na inter-relação com a saúde, isso denotaria maior distanciamento.

Em relação aos limites da atuação em equipe, Saporetti, Miranda e Belisário (2016) citam algumas dificuldades que os PEF's 
Inserção e atuação do profissional de educação física nos núcleos de apoio à saúde... Luciana Maria de Sobral • Rogério Cruz de Oliveira • Ricardo José Gomes • Camila Aparecida Machado de Oliveira

enfrentam cotidianamente em sua atuação, destacando-se a não compreensão do papel do PEF perante os demais colegas, encontrando eco em nosso estudo. Aqui, é perceptível a questão da legitimidade dos PEF's no campo da produção de cuidado em saúde. Para Furtado e Knuth (2015, p.519),

[...] um entendimento sobre a atuação da EF no contexto do NASF é como dar um passo adiante a fim de conquistar um espaço, uma identidade e, sobretudo, um trabalho valorizado e reconhecido por tratar-se de uma realidade ainda desconhecida, tanto pelos outros profissionais, quanto pela gestão.

Ou seja, mesmo possuindo legalidade de atuação, a relevância da EF no NASF e no SUS ainda parece ser um terreno a ser conquistado junto à equipe, gestão e munícipes, não sendo tarefa simples e circunscrita apenas aos PEF's, mas envolta pelas políticas de formação, valorização social e relevância acadêmica, principalmente na direção da superação de que a EF (sozinha) produz saúde por meio da atividade física.

No que tange às atividades desenvolvidas, os dados aqui apresentados vão ao encontro de Souza e Loch (2011) e Saporetti, Miranda e Belisário (2016), em que as PCAF, na maioria dos grupos, são de exercícios aeróbios (caminhadas), alongamentos e exercícios resistidos. As aulas são ministradas no modelo tradicional, no qual se inicia com um aquecimento, parte principal, e termina com volta calma. Para Borges e Oliveira (2020), que investigaram a dinâmica das aulas de práticas corporais numa UBS do município de Santos-SP por meio de observações participantes, esse é um método clássico do treinamento esportivo que se distancia dos pressupostos do NASF e da EF no SUS. Entretanto, os autores concluíram que, mesmo a atuação estando enrijecida metodologicamente pelo treinamento esportivo, as aulas não deixaram de ser palco de encontro e sociabilidade, comungando assim para uma atenção integral. Frente ao exposto, faz-se necessário afirmar que a observação participante empreendida por Borges e Oliveira 
Inserção e atuação do profissional de educação física nos núcleos de apoio à saúde... Luciana Maria de Sobral • Rogério Cruz de Oliveira • Ricardo José Gomes • Camila Aparecida Machado de Oliveira

(2020) permite compreender o fenômeno por uma perspectiva mais abrangente (in loco), possibilitando captar as minúcias do objeto de estudo, que, no caso do nosso estudo, estiveram limitadas pelas entrevistas.

Entretanto, observando os objetivos dos grupos de PCAF, também foi possível compreender uma prática pedagógica emancipatória, situada na amplitude de finalidades. Em síntese, pode-se afirmar que os PEF's de Santos-SP atuam no horizonte da promoção e prevenção da saúde em consonância com as diretrizes do NASF, que preveem

\begin{abstract}
[...] como essencial para a atuação do profissional de saúde o reconhecimento da promoção da saúde como construção gerada nessa dinâmica de produção da vida [...] prevenção e humanização da saúde, com diferentes formatos em sua execução, podendo se apresentar como política transversal ou articuladora, dentro de uma matriz de princípios norteadores das práticas de saúde local (BRASIL, 2010, 124).
\end{abstract}

Em um estudo realizado por Ferreira, Diettrich e Pedro (2015), que pesquisou a influência da prática de atividade física sobre a qualidade de vida em usuários do SUS, foi constatado que, de acordo com os 8 domínios previstos no SF-365, os usuários que praticam atividade física apresentaram maiores scores de qualidade de vida do que os que não praticavam. De acordo com os autores, tais dados atendem um dos objetivos da Portaria 154/2008, que “[...] prevê a prática de atividade física como uma de suas ações, considerando que ela deve propiciar melhoria da qualidade de vida da população, entre outros" (FERREIRA, DIETTRICH e PEDRO, 2015, p.797). Putzel (2011), que avaliou a percepção dos próprios usuários que participavam de grupos de atividade física nas UBS, afirmou que houve melhorias tanto fisiológicas quanto psicossociais, consequentemente influenciando numa melhor qualidade

\footnotetext{
50 questionário SF - 36 é um instrumento genérico para avaliação da qualidade de vida formado por 36 itens englobados por 8 escalas ou componentes: capacidade funcional, aspectos físicos, dor, estado geral da saúde, vitalidade, aspectos sociais, aspectos emocionais e saúde mental (CICONELLI et al., 1999 citado em FERREIRA, DIETTRICH e PEDRO, 2015).
} 
Inserção e atuação do profissional de educação física nos núcleos de apoio à saúde... Luciana Maria de Sobral • Rogério Cruz de Oliveira • Ricardo José Gomes • Camila Aparecida Machado de Oliveira

de vida. Embora não mencionado pelos PEF's desse estudo, a qualidade de vida se espraia também como horizonte de intervenção, principalmente quando é mencionada a autonomia do usuário como objetivo.

Em relação ao processo de avaliação, percebe-se que é ainda um ponto que carece de investimento e estruturação, pois compreende-se que uma anamnese tem a sua importância, mas não pode ser a única via do processo avaliativo. Para Loch, Dias e Rech (2019, p.4-5),

\begin{abstract}
[...] os PEF devem ter alguma forma de avaliação de sua prática profissional. Evidentemente, a avaliação deve ser entendida de uma forma ampla, como parte importante de um processo e fundamental para proporcionar informações para uma melhor tomada de decisão. Ademais, destaca-se que estas avaliações não devem ser apenas focadas na aptidão física de pessoas que participam dos grupos formais. Sugere-se a inclusão de outras variáveis como por exemplo: comportamentos relacionados à saúde, participação social, qualidade de vida, atitudes, autoeficácia, conhecimento etc.
\end{abstract}

Em síntese, a avaliação é nevrálgica em qualquer processo educativo. No caso específico desse estudo, a limitação, ou sua ausência, cria barreiras sobre a clareza do alcance dos objetivos previstos pelos PEF's e pela equipe, bem como sobre novas demandas e necessidades em saúde da população, pois toda avaliação acaba por alimentar o planejamento de novas intervenções.

Nesse sentido, acredita-se que a prática pedagógica tradicional dos PEF's no NASF de Santos-SP só será completamente suplantada por uma prática pedagógica emancipatória quando se superar o limite da avaliação, que, sem correspondência com o objetivo - seu par dialético (FREITAS, 1995) -, dificulta a clareza no cuidado à saúde dos munícipes. Por outro lado, a convivência, a melhoria das condições da vida diária e o desenvolvimento da autonomia, presentes nos discursos dos PEF's relacionados aos ob- 
Inserção e atuação do profissional de educação física nos núcleos de apoio à saúde...

Luciana Maria de Sobral • Rogério Cruz de Oliveira • Ricardo José Gomes • Camila Aparecida Machado de Oliveira

jetivos, são potenciais caminhos de uma atuação mais articulada com os ideários do NASF. Em síntese, seria possível afirmar que se trata de uma prática pedagógica instrumental aberta aos ideários críticos. Ou, como afirmaram Borges e Oliveira (2020), um modelo tradicional de atuação com ares emancipatórios.

\section{Conclusão}

Conclui-se que os PEF's, que atuam em equipes NASF do município de Santos-SP, foram inseridos nesse contexto pela condição de trabalho que exerciam na SMS há uma década. Nesse sentido, embora seja uma atribuição desafiadora, enxergam-na como um avanço na produção de cuidado em saúde dos munícipes.

No que tange à atuação, os PEF's oscilam entre uma prática pedagógica tradicional, envolta por conteúdos e métodos clássicos do treinamento esportivo, e uma prática pedagógica emancipatória, a qual se ocupa da convivência, sociabilidade, condições de vida e autonomia dos munícipes. Tal fato nos permite afirmar que, embora haja um potencial alinhamento da atuação em relação às diretrizes do NASF no município, existem clássicos limites que ainda precisam ser superados, principalmente na direção da ampliação do número de PEF's, legitimidade frente à equipe multiprofissional e um investimento na implantação de um processo avaliativo que corresponda à complexidade do fazer saúde em EF na ABS.

\section{Referências}

ASSIS, M.M.A. et al. Dimensões teóricas e metodológicas da produção do cuidado em saúde. In: ASSIS, M.M.A et al. (Orgs.). Produção do cuidado no Programa Saúde da Família: olhares analisadores em diferentes cenários. Salvador: EDUFBA, 2010. p.13-38. 
Inserção e atuação do profissional de educação física nos núcleos de apoio à saúde... Luciana Maria de Sobral • Rogério Cruz de Oliveira • Ricardo José Gomes • Camila Aparecida Machado de Oliveira

BORGES, E.O.; OLIVEIRA, RC. A dinâmica das aulas de práticas corporais numa unidade básica de saúde do município de SantosSP. Pensar a Prática, Goiânia, v.23, s/n, p.e57313, 2020. BRACHT, V. Educação física e aprendizagem social. Porto Alegre: Magister, 1992.

BRASIL. Resolução n.6, de 18 de dezembro de 2018. Institui Diretrizes Curriculares Nacionais dos cursos de graduação em Educação Física e dá outras providências. Diário Oficial da República Federativa do Brasil, Poder Executivo, Brasília, DF, 19 dez. 2018a.

BRASIL. Resolução n.569, de 19 de janeiro de 2018. Princípios gerais para as Diretrizes Curriculares Nacionais dos cursos de graduação da área de Saúde. Diário Oficial da República Federativa do Brasil, Poder Executivo, Brasília, DF, 26 fev. 2018b. BRASIL. Ministério da Saúde. Núcleo de Apoio à Saúde da Família: Ferramentas para a gestão e para o trabalho cotidiano. Caderno de Atenção Básica, Brasília, v. 1, n. 39, 2014.

BRASIL. Ministério da Saúde. Política Nacional da Atenção

Básica. Secretaria de Atenção à Saúde, Brasília, 2012.

BRASIL. Ministério da Saúde. Diretrizes do NASF: Núcleo de Apoio Saúde da Família. Caderno de Atenção Básica - Série A. Normas e Manuais Técnicos, Brasília, n. 27, 2010.

BRASIL. Ministério da Saúde. Portaria 154, de 24 de janeiro de 2008. Diário Oficial da República Federativa do Brasil, Poder Executivo, Brasília, DF, 04 fev. 2008.

BUENO, A.X.A Educação Física na saúde: reflexões acerca do fazer da profissão no SUS. In: WACHS, F.; ALMEIDA, U.R.; BRANDÃO, F.F.F. (Orgs). Educação Física e saúde coletiva: cenários, experiências e artefatos culturais. Porto Alegre: Rede UNIDA, 2016. p.145-168.

CARVALHO, Y.M. Promoção da Saúde, Práticas corporais e Atenção Básica. Revista Brasileira Saúde da Família, Brasília, n.11, p.33-45. 2006. 
Inserção e atuação do profissional de educação física nos núcleos de apoio à saúde... Luciana Maria de Sobral • Rogério Cruz de Oliveira • Ricardo José Gomes • Camila Aparecida Machado de Oliveira

CAMPOS, C.J.G. Método de análise de conteúdo: ferramenta para a análise de dados qualitativos no campo da saúde. Revista Brasileira de Enfermagem, Brasília, v.57, n.5, p.611-614, 2004. FERREIRA, J.S.; DIETTRICH, S.H.C.; PEDRO, D.A. Influência da prática de atividade física sobre a qualidade de vida de usuários do SUS. Saúde e Debate, Rio de Janeiro, v.39, n.106, p.792-801, 2015.

FONSECA, S.A. et al. Nota preliminares sobre a Associação Brasileira de Ensino da Educação Física para a Saúde - ABENEFS. Caderno FNEPAS, Rio de Janeiro, v. 2, s/n, p.38-48, 2012.

FREITAS, L. C. de. Crítica da organização do trabalho pedagógico e da didática. Campinas: Papirus, 1995.

FURTADO, G.V.N.; KNUTH, A.G. Núcleo de Apoio à Saúde da Família (NASF) em Rio Grande/ RS: percepções sobre o trabalho realizado pela educação física. Revista Brasileira de Atividade Física e Saúde, Florianópolis, v.20, n.5, p.514-523, 2015. KOKUBUN, E. et al. Programa de Atividade Física em Unidade Básica de Saúde: Relato de Experiência no município de Rio Claro - SP. Revista Brasileira de Atividade Física e Saúde, Florianópolis, v.12, n.1, p. 45-53, 2007.

KOUMANTAREAS, J.; OLIVEIRA, R.C. Sports and health from the perspective of physical education professionals. Motriz, Rio Claro, v.24, n.4, p.e101870, 2018.

KUNZ, E. Transformação didático-pedagógica do esporte. ljuí: Unijuí, 1994.

LOCH, M. R.; DIAS, D.F.; RECH, C.R. Apontamentos para a atuação do Profissional de Educação Física na Atenção Básica à Saúde: um ensaio. Revista Brasileira de Atividade Física e Saúde, Florianópolis, v.24, s/n, p.1-5, 2019.

MAGALHÃES JUNIOR, H.M. Redes de Atenção à Saúde: rumo à integralidade. Divulgação em saúde para debate, Brasil, v.52, s/n, p.15-33, 2014. 
Inserção e atuação do profissional de educação física nos núcleos de apoio à saúde... Luciana Maria de Sobral • Rogério Cruz de Oliveira • Ricardo José Gomes • Camila Aparecida Machado de Oliveira

MINAYO, M.C.S. O desafio do conhecimento: pesquisa qualitativa em saúde. 14.ed. São Paulo: Hucitec, 2014.

MINAYO, M.C.S. Ciência, técnica e pesquisa: o desafio da pesquisa social. In: MINAYO, M.C.S. (Org.). Pesquisa Social: Teoria, método e criatividade. 9 ed. Petrópolis: Vozes, 1994. p.9-30.

OLIVEIRA, R.C.; ANDRADE, D.R. Formação profissional em Educação Física para o setor da saúde e as Diretrizes Curriculares Nacionais. Pensar a Prática, Goiânia, v.19, n.4, p.721-733, 2016.

PUTZEL, M. P. Efeitos da atividade física no SUS: Percepções de usuários e profissionais da saúde. 2011. 77f. Monografia (Especialização em Educação Física)-Universidade Comunitária da Região de Chapecó, Chapecó, 2011.

SANTOS, S.F.S; BENEDETTI, T.R.B. Cenário de implantação do Núcleo de Apoio a Saúde da Família e a inserção do profissional de Educação Física. Revista Brasileira de Atividade Física e Saúde, Florianópolis, v.17, n.3, p.188-194, 2012.

SAPORETTI, G.M.; MIRANDA, P.S.C.; BELISÁRIO, S.A. O Profissional de Educação Física e a Promoção da Saúde em Núcleos de Apoio à Saúde da Família. Trabalho, Educação e Saúde, cidade, Rio de Janeiro, v.14, n.2, p.523-543. 2016.

SCHUH, L.X. et al. A inserção do profissional de educação física nas equipes multiprofissionais da estratégia saúde da família. Saúde, Santa Maria, v.41, n.1, p.29-36, 2015.

SOUZA, S.C.; LOCH, M.R. Intervenção do profissional de educação física nos Núcleos de Apoio à Saúde da Família em municípios do norte do Paraná. Revista Brasileira de Atividade Física e Saúde, Florianópolis, v. 16, n. 12, p.5-10, 2011.

TRIVIÑOS, A. N. S. Introdução à pesquisa em ciências sociais: A pesquisa qualitativa em educação. 16.reimp. São Paulo: Atlas, 2008. 
Inserção e atuação do profissional de educação física nos núcleos de apoio à saúde...

Luciana Maria de Sobral • Rogério Cruz de Oliveira • Ricardo José Gomes • Camila Aparecida Machado de Oliveira

\section{Publisher}

Universidade Federal de Goiás. Faculdade de Educação Física e Dança. Publicação no Portal de Periódicos UFG. As ideias expressadas neste artigo são de responsabilidade de seus autores, não representando, necessariamente, a opinião dos editores ou da universidade. 